\title{
DNA Methylation Changes in Endometrium and Correlation with Gene Expression During Ovarian Hyper-stimulation
}

\section{Bo Sun}

Zhengzhou University First Affiliated Hospital

\section{Yujia Ma}

Zhengzhou University First Affiliated Hospital

\section{Fang Wang}

Zhengzhou University First Affiliated Hospital

\section{Linli Hu}

Zhengzhou University First Affiliated Hospital

\section{Shanjun Dai}

Zhengzhou University First Affiliated Hospital

\section{Yile Zhang}

Zhengzhou University First Affiliated Hospital

\section{Yingpu Sun ( $\nabla$ syp2008@vip.sina.com )}

Zhengzhou University First Affiliated Hospital

\section{Research}

Keywords: Endometrium, Transcriptome, DNA methylation, Controlled ovarian hyper-stimulation ( $\mathrm{COH})$, Endometrial receptivity

Posted Date: August 28th, 2020

DOI: https://doi.org/10.21203/rs.3.rs-64858/v1

License: (c) (1) This work is licensed under a Creative Commons Attribution 4.0 International License. Read Full License 


\section{Abstract}

Persistent supraphysiological serum estradiol (E2) levels during controlled ovarian hyper-stimulation $(\mathrm{COH})$ have a detrimental effect on endometrial receptivity. In this study, we explored RNA expression and DNA methylation profiles from patients' endometrium. The patients were divided into two groups: the $\mathrm{COH}$ cycle $(n=3, h C G+7)$ group and normal cycle group $(n=3, L H+5)$. Quantitative RT-PCR was used to validate the expression of selected differentially expressed genes (DEGs). Comparing natural and stimulated endometrium transcriptome profiles revealed 640 DEGs, with a $>2$-fold change (FC) and $p<$ 0.01 . The DEGs were reported to be involved in endometrial receptivity and epithelial-mesenchymal transition (EMT). The expression of IGFBP-1, MMP9, FGF9, LIF, WNT4, HAND2, and immune systemrelated genes were significantly up-regulated. By clustering and KEGG pathway analysis, molecules and pathways associated with endometrial receptivity (PI3K-Akt signaling pathway) were identified. DNA methylation was partially correlated to gene expression. In conclusion, RNA-seq $\mathrm{COH}$ affected endometrial receptivity and EMT/MET process by accelerated the decidualization process and broken the balance of estrogen and progesterone receptors expression. However, this was not associated with changes in DNA methylation.

\section{Introduction}

Controlled ovarian hyperstimulation $(\mathrm{COH})$ is a technique widely used in vitro fertilization (IVF). Highquality embryos and a receptive endometrium are significant during $\mathrm{COH}$. In recent years, several $\mathrm{COH}$ protocols have been adopted to optimize the quantity and quality of embryo transferred and improve on subsequent pregnancy success rate. However, even with a high-quality embryo, implantation failure can occur due to insufficiency of the endometrium deficiency and this is a major cause of pregnancy failure. Previous human studies [1, 2] have shown that the supraphysiological hormonal levels, especially serum estradiol (E2) impair endometrial receptivity or displace the window of implantation (WOI) affecting embryo adhesion [3]. In vitro studies have also confirmed that increasing E2 levels are deleterious to embryonic implantation because they directly affect the embryo [4] and endometrium [5].

Endometrial alterations in clinical practice are characterized by the endometrial thickness and morphological alterations which require further histological [6] and biochemical investigations [7]. Recently, researchers have focused on omics analysis of changes in the endometrium during $\mathrm{COH}$. In stimulated cycles, progesterone receptor (PGR) and related pathways are reduced during WOI, while estrogen receptor (ER) and related pathway are increased accordingly [8-11]. In natural as well as stimulated cycles, the majority of the modulated genes during endometrial receptivity are reported to be up-regulated. Besides, the genes involved in the implantation process are absent in $\mathrm{COH}$ cycles [12-14]. Studies have reported that the endometrial genomic profile in the presence of a $\mathrm{GnRH}$ antagonist protocol is more similar to the natural cycle compared to that treated with a $\mathrm{GnRH}$ agonist protocol [15-17]. Therefore, there is need to optimize $\mathrm{COH}$ protocols to improve endometrial receptively. 
DNA methylation (DNAm), as one of the most common forms of epigenetic modification. Methylation profiles in human endometrium also change across the menstrual cycle with thousands of genes differentially methylated between cycle stages [18]. A comparison of the changes in transcriptomes and corresponding DNAm from the same samples showed an association of DNA methylation and gene expression [18]. Genes involved in cell adhesion, immune response and developmental processes are the most affected by aberrant methylation changes during epithelial-mesenchymal transition $(E M T) /$ mesenchymal-epithelial transition (MET) regulation processes $[19,20]$. Previous studies have shown that DNA hyper-methylation has a detrimental effect on endometrial receptivity in the presence of high progesterone (P4) levels during $\mathrm{COH}$ [21]. E2 and P4 induced decidualization of human endometrial stromal cell in vitro has been shown to exhibit DNAm changes $[18,22]$. However, the status of DNAm profiles in supraphysiological E2 levels during $\mathrm{COH}$ and their associations with changes in gene expression remain unknown.

In the present study, we explored the effect of supraphysiological levels of serum E2 on DNAm profiles during $\mathrm{COH}$ and correlate with gene expression profiles of endometrial receptivity.

\section{Material And Methods}

\section{Patient characteristics and tissue collection.}

Endometrial samples ( $3 \mathrm{COH}$ and 3 normal cycle specimens) were obtained from 6 healthy infertile patients. Inclusion criteria included age range between $25-35$ years, BMI between $18-25 \mathrm{~kg} / \mathrm{m}^{2}$ and a regular menstruation cycle of 21-35 days. In the NOR group, tissues were obtained from the patients at their mid secretory phase $(\mathrm{LH}+5)$ of the natural cycle and the patients had not been on hormonal treatment for at least three months before tissue sampling. For the $\mathrm{COH}$ group, gonadotrophin-releasing hormone agonist ( $\mathrm{GnRH}-\mathrm{a})$ and $\mathrm{Gn}$ were used for ovarian stimulation. Oocyte retrieval was performed 36$37 \mathrm{~h}$ after HCG administration by the transvaginal ultrasound approach. Insemination was carried out 4 to 5 hours after ovum pick-up (OPU). About 16 to 18 hours after insemination, the presence of pro-nuclei was detected and freezing of all embryos performed. Patients were diagnosed with ovarian hyperstimulation syndrome (OHSS) and after 5 days of OPU (hCG+7), their endometrium was collected. Endometrial biopsies were collected with Pipelle catheters under sterile conditions from the uterine fundus. A portion of the collected endometrial tissue was snap-frozen in liquid nitrogen and stored at -80 ${ }^{\circ} \mathrm{C}$ in RNAlater (Ambion Inc., Austin, TX, USA) until further processing. Tissue collection was approved by the Ethics Review Committee of the University of Zhengzhou, and written informed consent was obtained from all participants.

\section{RNA extraction and expression analysis.}

Total RNA was extracted from endometrial tissues using the TRIzol reagent (Invitrogen, Carlsbad, CA) according to the manufacturer's instructions. The RNA quality was determined using a NanoDrop 2000 
Spectrophotometer (ThermoFisher, USA). Total RNA extracted was used for cDNA library construction as described in Supplemental Figure 1 and sequencing performed using Illumina $\mathrm{HiSeq}^{\mathrm{TM}} 2500$ (Gene Denovo Biotechnology Co., Guangzhou, China). All the high-quality reads were mapped using Bowtie2 [23] to ribosomal RNA (rRNA). All rRNA reads were mapped to the human genome (GRCh38/hg38) using TopHat2 (version 2.0.3.12) [24]. Cufflinks [25] and Cuffmerge were used to reconstruct and merge the transcripts.

Principal component analysis (PCA) was performed with R package gmodels (http://www.r-project.org/). Differentially expressed genes (DEGs) were identified using edgeR with a fold change $(F C)>2$ and a false discovery rate $(F D R)<0.05$. The $D E G s$ were then subjected to enrichment analysis including Gene Ontology (GO) and the Kyoto Encyclopedia of Genes and Genomes (KEGG) pathway analysis.

Quantitative real-time PCR was used to validate the RNA sequencing (RNA-seq) results. Synthesis of cDNA from extracted total RNA (2ug) was performed with a Fast Quant RT kit (TIANGEN, China) according to the manufacturer's instructions. Real-time PCR was performed using the SYBR Green master mix (Vazyme, China) on a Step One Plus Real-Time PCR System (Applied Biosystems, USA). All quantitative PCR reactions were run in triplicate. Primer sequences are presented in Supplemental Table 1.

\section{DNA extraction and methylation analysis.}

Genomic DNA was isolated from endometrial tissue using Qiagen DNeasy Kits and quantified using PicoGreen assay. DNA concentration and integrity were assessed using a Nano Drop spectrophotometer and Agarose Gel Electrophoresis, respectively. DNA bisulfite sequencing libraries were prepared as described in Supplemental Figure 1. After filtering, the clean reads were mapped to the species reference genome using BSMAP software (version: 2.90). A custom Perl script was used to call methylated cytosine's which were tested with the correction algorithm as described in previous studies [26].

Methyl-kit software (V1.4.1) [27] was used for the analysis of differentially methylated regions (DMRs). To identify DMRs between two samples, the minimum read coverage to call a methylation status for a base was set to 4 . DMRs within a 200bp window for each sequence context was determined according to different criteria: 1) For $C G$ and $C H G$, the numbers in each window $\geq 5$, the absolute value of the difference in methylation ratio $\geq 0.25$, and $\mathrm{q} \leq 0.05 ; 2$ ) For $\mathrm{CHH}$, the numbers in a window $\geq 15$, the absolute value of the difference in methylation ratio $\geq 0.15$, and $q \leq 0.05 ; 3$ ) For all $C$, the numbers in a window $\geq 20$, the absolute value of the difference in methylation ratio $\geq 0.2$, and $q \leq 0.05$.

\section{Statistical analysis}

The data for patients in the $\mathrm{COH}$ and NOR groups were expressed as the mean \pm standard deviation or number (percentage). The Mann-Whitney $U$ test was used to study the continuous variable and non- 
normally distributed data were tested by Wilcoxon's rank-sum test. For the categorical variable data, we performed chi-squared test analysis or 2-way ANOVA was performed using SPSS software (version 21.0). ${ }^{*} \mathrm{P}<0.05$ was considered to be statistically significant.

\section{Results}

\section{Baseline parameters}

The baseline characteristics of the six patients are listed in Supplemental Table 2. Age, duration of fertility, body mass index, type of infertility and antral follicle count were comparable between the two groups. No statistically significant difference was reported in the basal hormone levels. Combined with the higher serum E2 concentration on the day of HCG $(4126.67 \pm 1293.18 \mathrm{pg} / \mathrm{mL}$ vs $103.33 \pm 53.63 \mathrm{pg} / \mathrm{mL}$, $\mathrm{p}=0.0058)$ and clinical symptoms after eggs removal, patients in $\mathrm{COH}$ group were hyper ovarian responders and they later developed OHSS. The lower serum LH concentrations on the day of HCG $(1.55 \pm 0.32$ vs $21.12 \pm 11.41 \mathrm{mlU} / \mathrm{mL}, \mathrm{p}=0.0411)$ resulted from the pituitary desensitization in $\mathrm{GnRH}$ agonist cycles.

\section{RNA-Seq data analysis}

Transcriptome analysis based on RNA-seq and PCA showed the clustering of the samples in each group (Figure 1A). There were 2796 DEGs ( $p$-value with FDR correction < 0.05), including 1870 upregulated and 926 downregulated genes in $\mathrm{COH}$ (Supplementary Data 1). Figure 1B shows volcano plots providing an overview of the differential status of gene expression.

GO and KEGG pathway analysis was performed on the DEGs. Enriched Go terms are showed in Figure 2A $B$ and arranged and displayed according to biological processes, cellular components, and molecular functions. For the cellular component annotation classification, both up- and down-regulated genes were mainly localized in the membranes, endomembrane system, vesicle, nucleus, and extracellular region. Concerning the molecular function, both up- and down-regulated genes were mainly associated with binding, catalytic activity, and signal transduction. The enriched biological processes for the up- and down-regulated genes were mainly involved in biological regulation, metabolic processes, response to stimulus and multicellular organismal process. KEGG pathway analysis showed that the top 20 KEGG pathways were significantly enriched and are shown in Figure $2 \mathrm{C}$. Among these pathways, the immune system (complement and coagulation cascades) and signal transduction (PI3K-Akt signaling pathway) were found to be the most enriched. Besides, the Ras signaling pathway, MAPK signaling pathway, and WNT signaling pathway were also enriched.

To further investigate the DEGs relationships, a co-expression network of 640 DEGs ( $p$-value $<0.01$ ) based on protein-protein interaction (PPI) is shown in Figure 3A. Four key genes, including C3, CXCL8, CXCL12, and PENK were considered to have important regulation and control ability. A total of 221 DEGs related to EMT, 83 DEGs involved in endometrial receptivity and 57 DEGs associated with both functions 
were screened (Figure 3B, Supplementary Data 2). The six up-regulated transcripts with the highest FC in EMT were MMP1, SOHLH2, ELOVL3, LBP, IL1B, and IGFBP1. The six down-regulated transcripts identified as receptivity-associated genes were NLRP5, SLC7A4, SEPRINB9, CD36, CREB3L1, and ATP6V0A4.

\section{DNAm profiling analysis}

Genome-wide DNAm profiles were studied by reduced-representation bisulfite sequencing (RRBS-Seq). The mean genome-wide methylation levels of the six samples in $\mathrm{C}, \mathrm{CG}, \mathrm{CHG}$, and $\mathrm{CHH}$ are shown in Supplementary Table 3. CG sites ( $\mathrm{mCG}$ ) modified by methylation were found to be stable and fully methylated. The methylated $\mathrm{CHH}$ sites $(\mathrm{mCHH})$ were unstable and were in a hemi-methylated or hypomethylated state.

The DMRs statistics between NOR and $\mathrm{COH}$ groups are shown in Figure 4A. The violin plots showing the distribution of methylation in each locus on 10 genes revealed significant differences in methylation (Figure 4B). The region-level analysis of all CG revealed 38 significant DMRs, of which 20 exhibited increased (associated with 10 genes) and 18 decreased (associated with 10 genes) methylation (Supplementary Table 4). The most significant DMRs included CG in the upstream region of RAP1B and the gene-body of NPAS3. RAP1B was also one of the genes highlighted in the site-level analysis (Supplementary Data 3).

GO and KEGG pathway analyses were performed to characterize the genes annotated to DMRs. In sitelevel analyses, no GO terms were significantly represented (Supplementary Data 4). This is typically mirrored by region-level analyses (Supplementary Data 5). Pathway enrichment analyses for the same gene lists showed enrichment in 12 pathways in site-level analysis, including insulin signaling, Rap1 signaling, renal cell carcinoma, neurotrophic signaling, N-Glycan biosynthesis, measles, Jak-STAT signaling, transcriptional misregulation in cancers, endocytosis, cytokine-cytokine receptor interaction, HTLV-I infection, PI3K-Akt signaling pathway. No enrichment was seen in region-level analysis; however, genes showing a correlation between methylation and gene expression were enriched for the Wnt signaling pathway.

\section{Correlation analysis}

Screening for gene function and expression and selected TSPAN4, NPAS3, RAP1B, and TGFB3 for qPCR was performed to evaluate the correlation between DNAm and transcriptome (Supplementary Figure 2). In this study, there was a limited correlation between methylation and transcriptome during $\mathrm{COH}$.

\section{Discussion}

The quality of endometrial receptivity during the WOI or the change of endometrial development time is highly implicated in implantation rates. Over the last decades, endometrial receptivity and EMT/MET 
processes have been widely studied. However, the detailed molecular mechanism during $\mathrm{COH}$ cycles remains unclear. Supraphysiological hormonal treatment in IVF cycles has shown a considerable impact on the molecular alterations in the endometrium. The main changes of molecules are focused on the endometrial glandular and stromal cells secreting and differentiating [28]. Elevated serum E2 levels in $\mathrm{COH}$ cycles are associated with increased glandular-stromal dyssynchrony and defective induction of PGR and a considerable decline in the number of cytosolic PGRs [3]. Besides, an increase in E2 and P4 causes endometrium transfer from the proliferative phase to the secretory phase and this leads to the WOI opening. Stromal cell decidualization reduces endometrial receptivity. High E2 levels limit the development of pectin but stimulate hyperplasia of endometrial collagen fibers, which can cause receptivity decline and implantation failure. However, with omics detection technology, it is possible to accurately and reliably assess the endometrium in clinical routine practice. Therefore, this study investigated the RNA expression and DNAm profile in patients who underwent the $\mathrm{COH}$ cycle in comparison with the natural cycle.

\section{Estrogen signaling pathway}

E2 promotes endometrial proliferation and regeneration in the natural cycle. The expression of ER1 occurs in the proliferative phase and ER2 is highly expressed in the secretory phase. E2 binds to nuclear ER (nER) which increases transcription and is involved in cell cycle genes (Cyclin B1/D1, CDK1/4), insulinlike growth factor 1 (IGF-1) and Ras/mitogen-activated protein kinase (MAPK) pathway-related genes [29, 30]. ER1, IGF1 partly upregulates epithelial proliferation which induces the PI3K/AKT pathway [31, 32]. The cAMP/PKA pathway which is triggered by G protein-coupled ER (GPER) inhibits AKT signaling during decidualization. WNT/ß-catenin pathway which is another ER1 mediated early proliferative signaling is positively regulated by inhibition of GSK-3 $B$ [29].

In this study, ER1 was reported to be significantly decreased and ER2 increased in the $\mathrm{COH}$ group compared with the natural group. E2 mediated epithelial proliferation occurred through the nuclear-and membrane-initiated steroid signaling. In the nuclear pathway, the down-regulation of ER1 was predominant. In the membrane pathway, E2 exerted its actions through a subpopulation of ER at the plasma membrane (mER) and GPER. Upon activation of these receptors, various signaling pathways were rapidly activated which ultimately influenced downstream transcription factors.

\section{PI3K/AKT signaling pathway}

The PI3K/AKT pathway was significantly enriched in various signal transduction pathways. The binding of growth factors (GF) to tyrosine kinase (RTK) receptors and $G$ protein-coupled receptors (GPCR) stimulated PI3K class IA and IB isoforms, respectively.PI3K catalyzed the production of phosphatidylinositol-3,4,5-triphosphate (PIP3) in the cell membrane. PIP3 served as a second messenger which activated AKT. AKT upregulated key cellular processes by phosphorylating substrates involved in protein synthesis, metabolism, and cell cycle. 


\section{RAS/MAPK signaling pathway}

E2 not only induces epithelial proliferation in the endometrium but also induces LIF in the glandular epithelium [33]. LIF action on the luminal epithelium regulates WNT/ß-catenin signaling, IGF1 signaling and FGF signaling [33]. LIF was significantly up-regulated in our results. FGF family are induced by E2 in the endometrial stroma [34]. As a potential paracrine mediator, FGF induces the RAS/MAPK and AKT pathway. In this study, part of the FGF family, particularly FGF9 was found to be up-regulated. Most of the E2-related pathways were overexpressed but the level of ER remained low. These findings suggested that proliferation-associated pathways were induced by E2. However, these findings should be verified using a larger sample size.

\section{WNT/ß-catenin signaling pathway}

The molecular protagonists in the decidualization route are P4 and cAMP [29]. The PGR-regulated genes and pathways include HOXA10, HAND2, IGFBP-1, and BMP2; ERK/MAPK, WNT/ß-catenin, and IHHCOUPTFIl pathways [35]. HOXA10 is a PGR target in the endometrium and has been reported to influence WNT4 expression around the implantation site in mice [36]. However, HOXA10 was not found in the DEGs, but the HOA family, HOXA5/6/7, HOXD8/9/10/11 were significantly up-regulated. Inhibitor DKK1 was found to be upregulated but did not block the WNT canonical pathway and this contributed to endometrial proliferation but not decidualization. Therefore, it is suggested that the decidualization process was partly impeded by the PGR-regulated pathway. The imbalance between ER and PGR related genes and pathways might impair endometrial receptivity.

\section{Complement and coagulation cascades}

Previous studies have confirmed that PRL, IGFBP-1, MMP9, and IL-6 are markers of decidualization [37]. Increased MMP secretion promotes endometrial stromal cells to support the decidualization process [38, 39]. In this study, both MMP9 and MMP2 were up-regulated suggesting promotion of the MET process. Decidualization is an example of the MET process and was found to be correlated with altered immune modulation and the complement cascade pathway [10]. Excessive E2 levels may be one of the reasons for maternal immune rejection due to the activation of the complement system. Furthermore, we established a co-expression network using the DEGs based on their significance and correlation. Both CXCL12 and its receptor CXCR4 were found to be upregulated and this confirmed that the proinflammatory response existed despite none embryo implantation. A large number of inflammatory responses inhibited endometrial decidualization and embryo implantation.

\section{DNA methylation}


Changes in DNAm during $\mathrm{COH}$ revealed 59 significant DMRs, out of which 23 exhibited increased and 36 decreased methylation between the two groups. In this study, no significant correlation between DNAm and transcriptome was reported. However, the methylation findings confirmed the dominance of the E2regulated pathway caused by $\mathrm{COH}$. Hence, it changed the endometrial receptivity and EMT process which mainly acted by P4.

\section{Conclusion}

In conclusion, RNA-seq $\mathrm{COH}$ had a deleterious effect on endometrial receptivity and EMT/MET process by advancing the decidualization process and affecting the balance between ER and PGR. However, these effects were not associated with changes in DNA methylation. This study has the following limitations; small sample size, single-center study, and retrospective design. Therefore, future prospective trials or meta-analyses are needed to confirm these findings.

\section{Clinical Perspectives}

- Supraphysiological serum E2 levels during $\mathrm{COH}$ is associated with embryo implantation and have an adversed effects on endometrial receptivity. However, the status of DNAm profiles in supraphysiological E2 levels during $\mathrm{COH}$ and their associations with changes in gene expression remain unknown.

- We confirmed that $\mathrm{COH}$ had a deleterious effect on endometrial receptivity and EMT/MET process, but these effects were not associated with changes in DNA methylation.

- Our results showing that the gene and DNAm change of endometrial during $\mathrm{COH}$ and to provide a theoretical basis of improving the endometrial receptively during IVF/ICSI clinical practices.

\section{Declarations}

\section{Ethics approval and consent to participate}

This study design was reviewed and approved by the Research Ethics Committee of the First Affiliated Hospital of Zhengzhou University.

\section{Consent for publication}

Not applicable.

\section{Availability of data and materials}

All data generated or analysed during this study are included in this published article and its supplementary information files. 


\section{Competing interests}

The authors declare that they have no competing interests.

\section{Author Contribution}

BS and YM collected the samples and wrote the initial draft. FW and LH edited the initial draft and supported in the statistical sense. SD and YZ critically reviewed the paper for important intellectual content. YS participated in the design of the study and revised the final version of the manuscript. All authors read and approved the final manuscript.

\section{Funding}

This work was funded by International (Regional) Cooperation and Exchange (ICE) Projects of the National Natural Science Foundation of China (NSFC) (FDN-81820108016); Chinese Medical Association Clinical Medical Research Special Fund Project (FDN-17020190688), the Medical Science and Technology Research Project Joint Co-construction Project of Henan Province (FDN-2018020116), and the Henan Provincial Higher Education Key Research Project Plan (FDN-19A320056).

\section{Acknowledgements}

This study was supported by the Reproductive Medicine Center at the First Affiliated Hospital of Zhengzhou University. We thank the people for their participation in this project.

\section{References}

1. Simon C, Cano D, Fau - F, Valbuena J, Valbuena D, Fau - Remohi A, Remohi J, Fau - Pellicer A, Pellicer. Clinical evidence for a detrimental effect on uterine receptivity of high serum oestradiol concentrations in high and normal responder patients, Hum Reprod, (1995).

2. Yu Ng EH, E Yeung Ws Fau - Yee Lan Lau, W.W. Yee Lan Lau E Fau - So, P.C. So Ww Fau - Ho, PC Ho, High serum oestradiol concentrations in fresh IVF cycles do not impair implantation and pregnancy rates in subsequent frozen-thawed embryo transfer cycles, Hum Reprod, (2000).

3. Montoya-Botero P, Polyzos NP, The endometrium during and after ovarian hyperstimulation and the role of segmentation of infertility treatment, Best Practice \& Research Clinical Endocrinology \& Metabolism, 33 (2019) 61-75.

4. Valbuena D, Martin JL, Fau - de Pablo J, J. de Pablo Jl Fau - Remohi, A. Remohi J Fau - Pellicer, C. Pellicer A Fau - Simon, C. Simon, Increasing levels of estradiol are deleterious to embryonic implantation because they directly affect the embryo, Fertil Steril, (2001). 
5. Ullah K, Rahman TU, Pan HT, Guo MX, Dong XY, Liu J, Jin LY, Cheng Y, Ke ZH, Ren J, Lin XH, Qiu XX, Wang TT, Huang HF, Sheng JZ. Serum estradiol levels in controlled ovarian stimulation directly affect the endometrium. J Mol Endocrinol. 2017;59:105-19.

6. Basir GS, O Ws Fau EH - Ng PC, Ng Eh Fau - Ho PC, Ho. Morphometric analysis of peri-implantation endometrium in patients having excessively high oestradiol concentrations after ovarian stimulation, Fertil Steril, 76 (2001).

7. Bhusane K, Bhutada S, Chaudhari U, Savardekar L, Katkam R, Sachdeva G. Secrets of Endometrial Receptivity: Some Are Hidden in Uterine Secretome. Am J Reprod Immunol. 2016;75:226-36.

8. Haouzi D, Dechaud H, Assou S, De Vos J, Hamamah S. Insights into human endometrial receptivity from transcriptomic and proteomic data. Reprod Biomed Online. 2012;24:23-34.

9. Garrido-Gomez T, Ruiz-Alonso M, Blesa D, Diaz-Gimeno P, Vilella F, Simon C. Profiling the gene signature of endometrial receptivity: clinical results. Fertil Steril. 2013;99:1078-85.

10. Altmae S, Koel M, Vosa U, Adler P, Suhorutsenko M, Laisk-Podar T, Kukushkina V, Saare M, VelthutMeikas A, Krjutskov K, Aghajanova L, Lalitkumar PG, Gemzell-Danielsson K, Giudice L, Simon C, Salumets A. Meta-signature of human endometrial receptivity: a meta-analysis and validation study of transcriptomic biomarkers. Sci Rep. 2017;7:10077.

11. Wang X, Yu Q. An update on the progress of transcriptomic profiles of human endometrial receptivity. Biol Reprod. 2018;98:440-8.

12. Horcajadas JA, Riesewijk A, Polman J, van Os R, Pellicer A, Mosselman S, Simon C. Effect of controlled ovarian hyperstimulation in IVF on endometrial gene expression profiles. Mol Hum Reprod. 2005;11:195-205.

13. Liu Y, Lee KF, Ng EH, Yeung WS, Ho PC. Gene expression profiling of human peri-implantation endometria between natural and stimulated cycles. Fertil Steril. 2008;90:2152-64.

14. Haouzi D, Assou S, Mahmoud K, Tondeur S, Reme T, Hedon B, De Vos J, Hamamah S. Gene expression profile of human endometrial receptivity: comparison between natural and stimulated cycles for the same patients. Hum Reprod. 2009;24:1436-45.

15. Mirkin S, Nikas G, Hsiu JG, Diaz J, Oehninger S. Gene expression profiles and structural/functional features of the peri-implantation endometrium in natural and gonadotropin-stimulated cycles. J Clin Endocrinol Metab. 2004;89:5742-52.

16. Simon C, Oberye J, Bellver J, Vidal C, Bosch E, Horcajadas JA, Murphy C, Adams S, Riesewijk A, Mannaerts B, Pellicer A. Similar endometrial development in oocyte donors treated with either highor standard-dose $\mathrm{GnRH}$ antagonist compared to treatment with a $\mathrm{GnRH}$ agonist or in natural cycles. Hum Reprod. 2005;20:3318-27.

17. Haouzi D, Assou S, Dechanet C, Anahory T, Dechaud H, De Vos J, Hamamah S. Controlled ovarian hyperstimulation for in vitro fertilization alters endometrial receptivity in humans: protocol effects. Biol Reprod. 2010;82:679-86.

18. Houshdaran S, Zelenko Z, Irwin JC, Giudice LC. Human endometrial DNA methylome is cycledependent and is associated with gene expression regulation. Mol Endocrinol. 2014;28:1118-35. 
19. Kukushkina V, Modhukur V, Suhorutsenko M, Peters M, Magi R, Rahmioglu N, Velthut-Meikas A, Altmae S, Esteban FJ, Vilo J, Zondervan K, Salumets A. T. Laisk-Podar, DNA methylation changes in endometrium and correlation with gene expression during the transition from pre-receptive to receptive phase. Sci Rep. 2017;7:3916.

20. Pathare ADS, Hinduja I. Aberrant DNA methylation profiling affecting the endometrial receptivity in recurrent implantation failure patients undergoing in vitro fertilization, Am J Reprod Immunol, (2019) e13196.

21. Xiong Y, Hu L, Zhang T, Wang M, Xu H, Li TC, Sun Y, Wang CC. Effects of high progesterone in in-vitro fertilization cycle on DNA methylation and gene expression of adhesion molecules on endometrium during implantation window. J Assist Reprod Genet. 2020;37:33-43.

22. Maekawa R, Tamura I, Shinagawa M, Mihara Y, Sato S, Okada M, Taketani T, Tamura H, Sugino N. Genome-wide DNA methylation analysis revealed stable DNA methylation status during decidualization in human endometrial stromal cells. BMC Genom. 2019;20:324.

23. Langmead B, Salzberg SL. Fast gapped-read alignment with Bowtie 2. Nat Methods. 2012;9:357-9.

24. Kim D, Pertea G, Trapnell C, Pimentel H, Kelley R, Salzberg SL. TopHat2: accurate alignment of transcriptomes in the presence of insertions, deletions and gene fusions. Genome Biol. 2013;14:R36.

25. Trapnell C, Roberts A, Goff L, Pertea G, Kim D, Kelley DR, Pimentel H, Salzberg SL, Rinn JL, Pachter L. Differential gene and transcript expression analysis of RNA-seq experiments with TopHat and Cufflinks. Nat Protoc. 2012;7:562-78.

26. Lister R, Pelizzola M, Dowen RH, Hawkins RD, Hon G, Tonti-Filippini J, Nery JR, Lee L, Ye Z, Ngo QM, Edsall L, Antosiewicz-Bourget J, Stewart R, Ruotti V, Millar AH, Thomson JA, Ren B, Ecker JR. Human DNA methylomes at base resolution show widespread epigenomic differences. Nature. 2009;462:315-22.

27. Akalin A, Kormaksson M, Li S, Garrett-Bakelman FE, Figueroa ME, Melnick A. C.E. Mason, methylKit: a comprehensive R package for the analysis of genome-wide DNA methylation profiles. Genome Biol. 2012;13:R87.

28. von Grothusen C, Lalitkumar S, Boggavarapu NR, Gemzell-Danielsson K, Lalitkumar PG. Recent advances in understanding endometrial receptivity: molecular basis and clinical applications. Am $J$ Reprod Immunol. 2014;72:148-57.

29. Makieva S, Giacomini E, Ottolina J, Sanchez A, Papaleo E, Viganò P. Inside the Endometrial Cell Signaling Subway: Mind the Gap(s). Int J Mol Sci. 2018;19:2477.

30. Marquardt RM, Kim TH, Shin JH, Jeong JW. Progesterone and Estrogen Signaling in the Endometrium: What Goes Wrong in Endometriosis?, Int J Mol Sci, 20 (2019).

31. Zhu L, Pollard JW. Estradiol-17beta regulates mouse uterine epithelial cell proliferation through insulin-like growth factor 1 signaling. Proc Natl Acad Sci U S A. 2007;104:15847-51.

32. Hewitt SC, Lierz SL, Garcia M, Hamilton KJ, Gruzdev A, Grimm SA, Lydon JP, Demayo FJ, Korach KS. A distal super enhancer mediates estrogen-dependent mouse uterine-specific gene transcription of Igf1 (insulin-like growth factor 1). J Biol Chem. 2019;294:9746-59. 
33. R. GX., S. CL., The Multifaceted Actions of Leukaemia Inhibitory Factor in Mediating Uterine Receptivity and Embryo Implantation, Am J Reprod Immunol., 75 (2016) 246-255.

34. Tsai SJ, Wu Mh Fau H-M - Chen P-C Chen Hm Fau - Chuang, L.-Y.C. Chuang Pc Fau - Wing, Wing LY, Fibroblast growth factor-9 is an endometrial stromal growth factor, Endocrinology., 143 (2002) 2715-2721.

35. Chi RA, Wang T, Adams N, Wu SP, Young SL, Spencer TE, DeMayo F. Human Endometrial Transcriptome and Progesterone Receptor Cistrome Reveal Important Pathways and Epithelial Regulators., J Clin Endocrinol Metab., (2019).

36. Daikoku T, Song H, Guo Y, Riesewijk A, Mosselman S, Das SK, Dey SK. Uterine Msx-1 and Wnt4 signaling becomes aberrant in mice with the loss of leukemia inhibitory factor or Hoxa-10: evidence for a novel cytokine-homeobox-Wnt signaling in implantation. Mol Endocrinol. 2004;18:1238-50.

37. Gellersen B, Brosens JJ. Cyclic decidualization of the human endometrium in reproductive health and failure. Endocr Rev. 2014;35:851-905.

38. Gellersen B, Reimann K, Samalecos A, Aupers S, Bamberger AM. Invasiveness of human endometrial stromal cells is promoted by decidualization and by trophoblast-derived signals. Hum Reprod. 2010;25:862-73.

39. Owusu-Akyaw A, Krishnamoorthy K, Goldsmith LT, Morelli SS. The role of mesenchymal-epithelial transition in endometrial function. Hum Reprod Update. 2019;25:114-33.

\section{Figures}
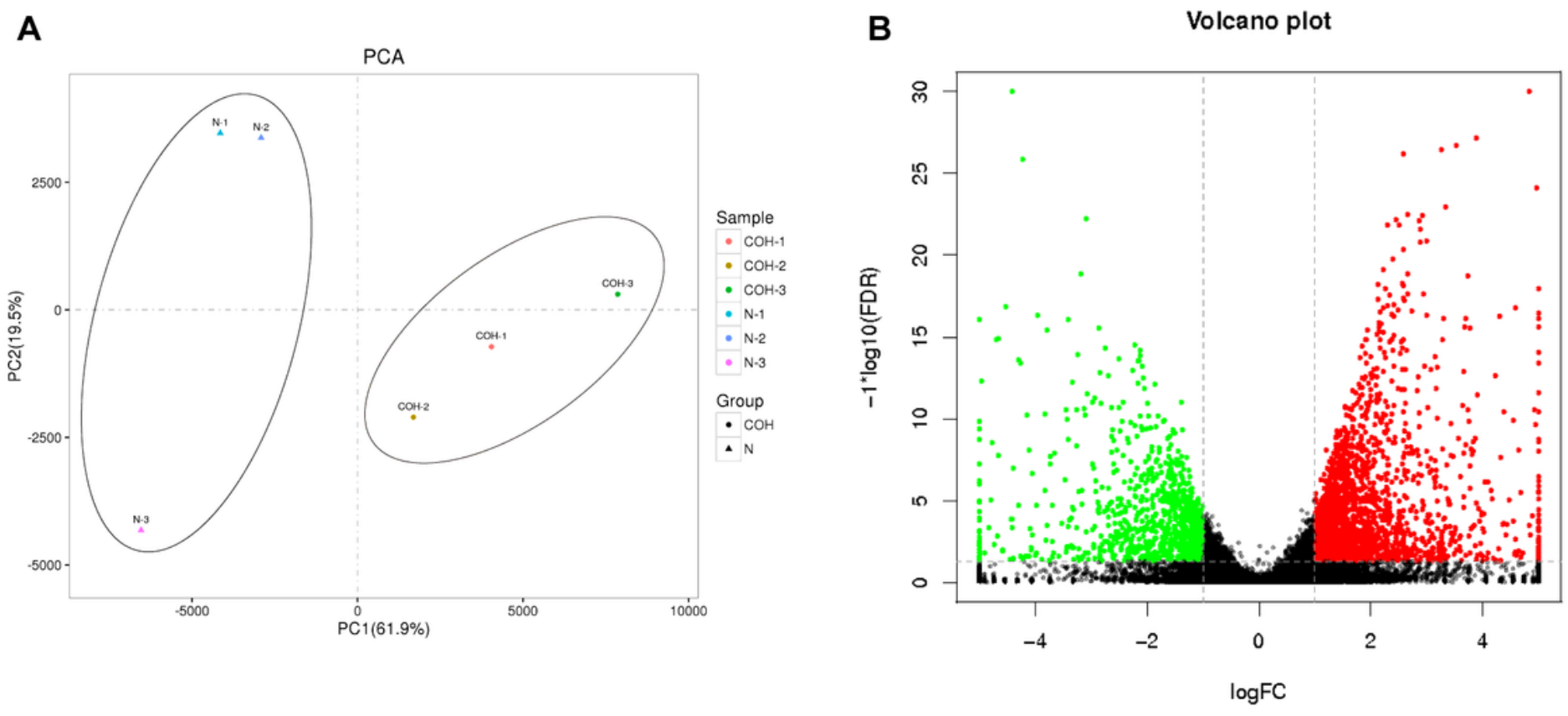

\section{Figure 1}


A)Transcriptome analysis based on RNA-seq and PCA showed the clustering of the samples in each group B) volcano plots providing an overview of the differential status of gene expression.

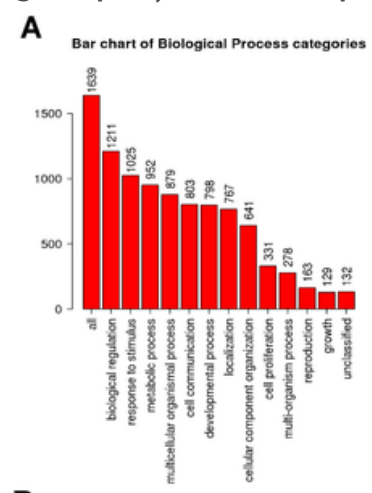

B

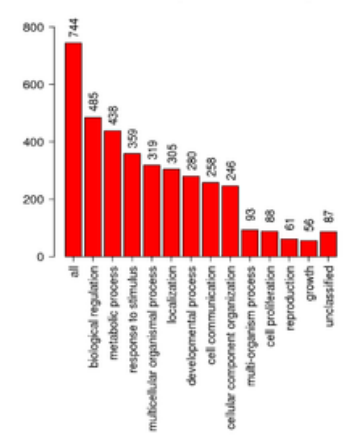

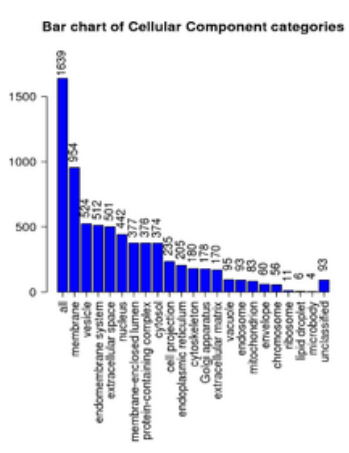
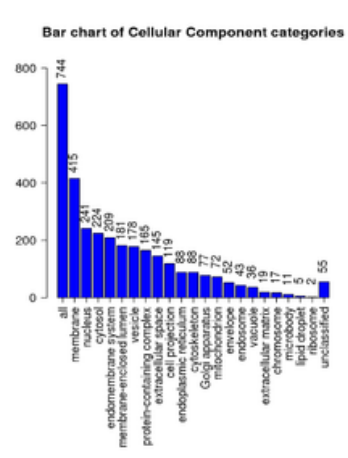

C
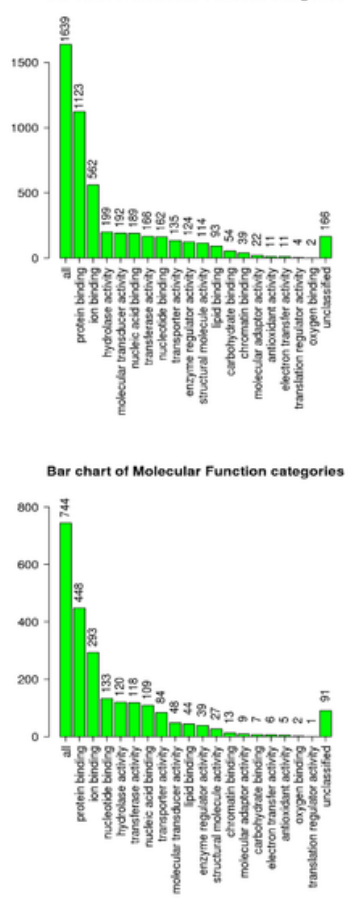

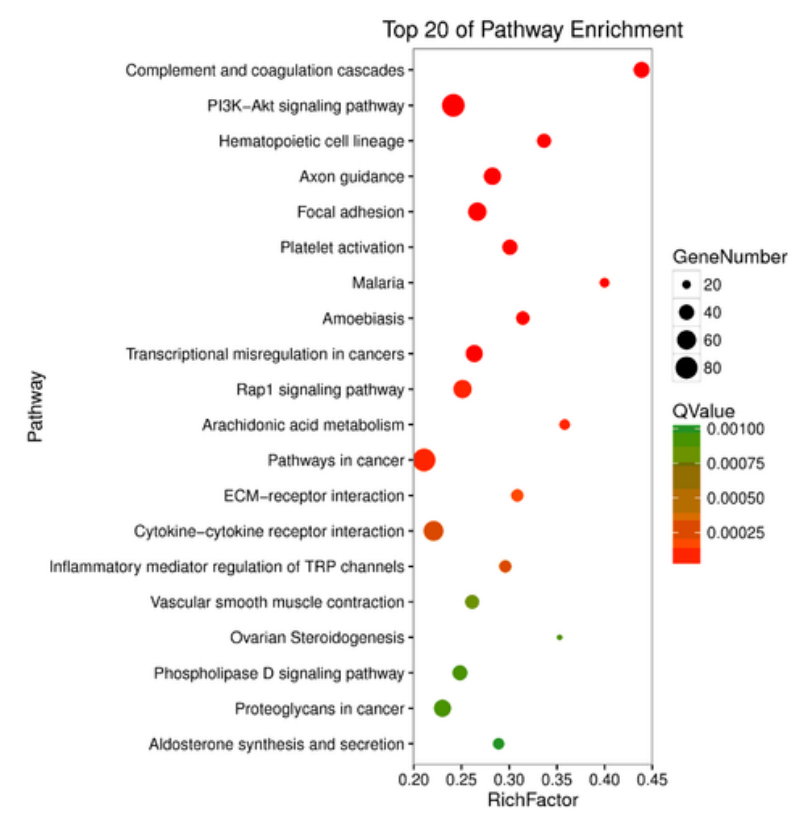

Figure 2

GO and KEGG pathway analysis was performed on the DEGs. Enriched Go terms are showed in $(A, B)$ KEGG pathway analysis showed that the top 20 KEGG pathways were significantly enriched (C)

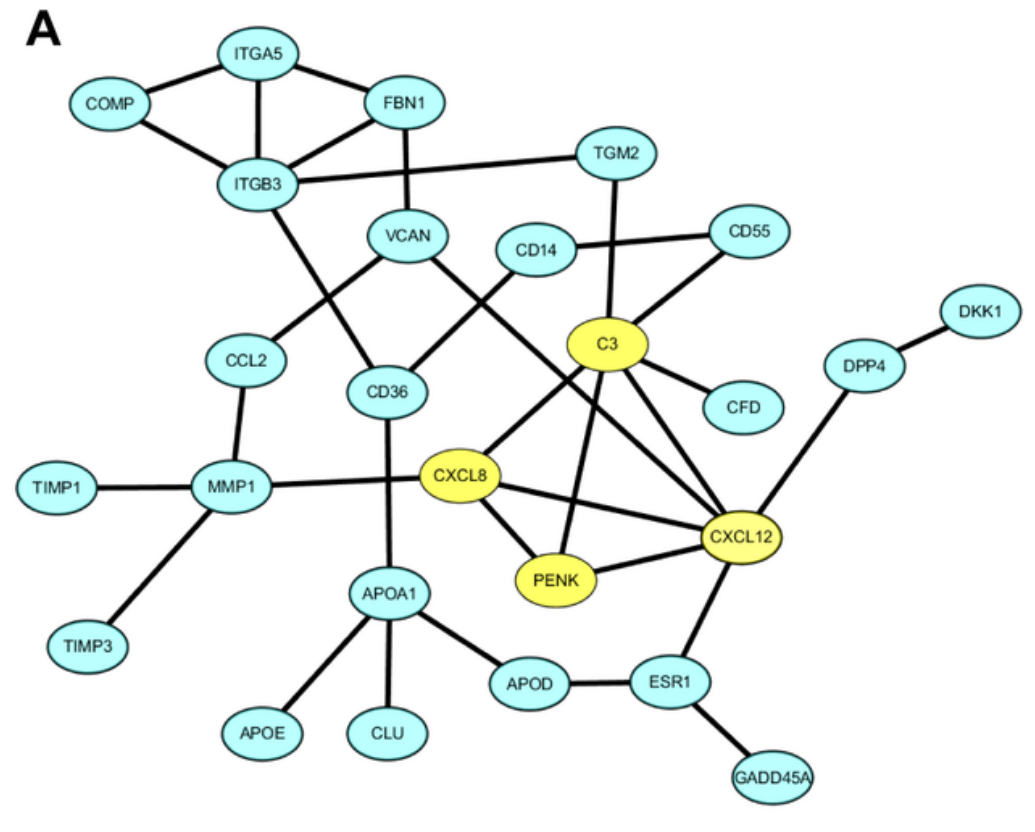

B

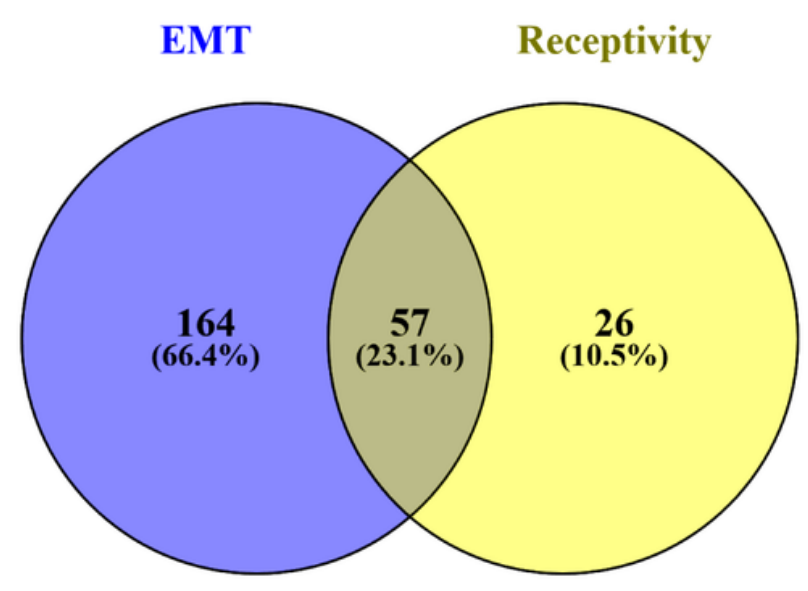

Figure 3

(A)a co-expression network of 640 DEGs (p-value < 0.01) based on protein-protein interaction (PPI) (B) A total of 221 DEGs related to EMT, 83 DEGs involved in endometrial receptivity and 57 DEGs associated 
with both functions

A

DMR Statistics

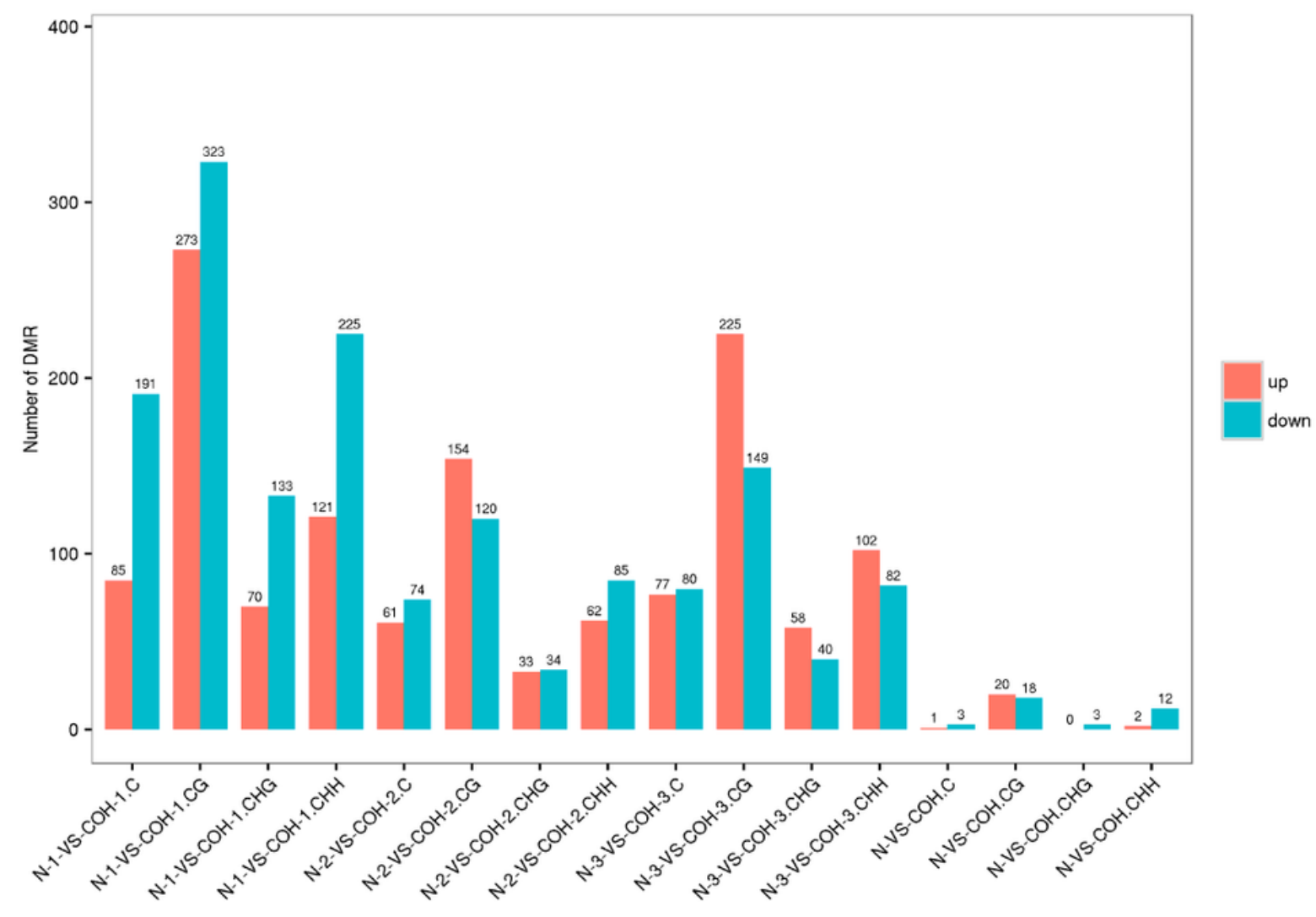

B
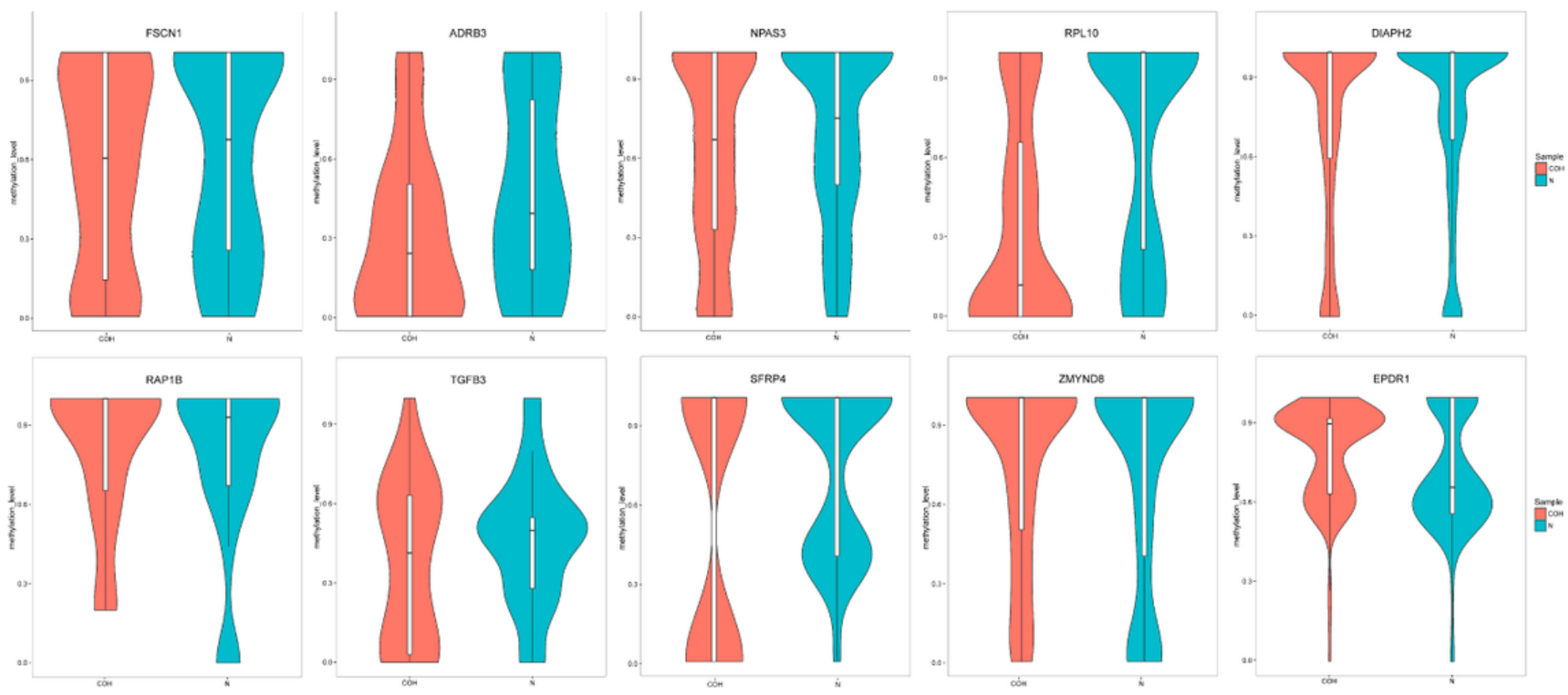

Figure 4

(A) The DMRs statistics between NOR and $\mathrm{COH}$ groups (B) The violin plots showing the distribution of methylation in each locus on 10 genes revealed significant differences in methylation

\section{Supplementary Files}


This is a list of supplementary files associated with this preprint. Click to download.

- SupplementaryData5.xlsx

- SupplementaryData4.xlsx

- SupplementaryData2.xlsx

- SupplementaryData3.xls

- SupplementaryData1.xlsx

- Onlinesupplementalfigure1.Png

- SupplementTable14.docx

- Onlinesupplementalfigure2.Png 